\title{
ACLIMATIZAÇÃO DE GENÓTIPOS DE PALMA FORRAGEIRA Opuntia stricta (Haw.) E Nopalea cochenilifera (L.) Salm-Dyck RESISTENTES A COCHONILHA-DO-CARMIM (Dactylopius opuntiae)
}

\author{
M. DE F. B. DUTRA ${ }^{1}$, M. H. I. ALOUFA ${ }^{2}$, N. F. DE MELO³, J. I. P. LEITE ${ }^{4}$ \\ Universidade Federal do Rio Grande do Norte ${ }^{1,2}$, Universidade Estadual de Feira de Santana ${ }^{3}$, Instituto Federal \\ de Educação Ciência e Tecnologia do Rio Grande do Norte 4 \\ ORCID ID: http://orcid.org/0000-0002-5923-0674 ${ }^{1}$ \\ mfbdutra@hotmail.com ${ }^{1}$
}

Submetido 07/07/2020 - Aceito 30/10/2020

DOI: $10.15628 /$ holos.2020.10689

\begin{abstract}
RESUMO
A irregularidade das chuvas limita a produção natural de alimentos para os animais no semiárido Nordestino, lança-se mão da palma forrageira como recurso alimentar estratégico, pois esta cactácea possui aspectos fisiológicos essenciais, viabilizando a economia e o cultivo por longos períodos de estiagem. O objetivo deste trabalho foi testar como diferentes tipos de substrato influenciam o crescimento das palmas orelha de elefante e miúda resistentes à cochonilha-do-carmim durante o processo de aclimatização. Fragmentos de cladódios das duas variedades de palma, miúda e orelha de elefante, foram cultivados em meio de cultura Murashige e Skoog (1962), contendo reguladores de crescimento, em seguida os frascos foram abertos, as mudas lavadas em água corrente e submetidas à aclimatização em estufa com
\end{abstract}

sistema de nebulização programada e temperatura constante de $30^{\circ} \mathrm{C}$ no qual foram testados quatro tipos de substrato: areia barrada, substrato orgânico comercial, areia barrada acrescida de esterco bovino e areia barrada acrescida de cama de frango. Após 120 dias de acompanhamento, o teste de análise de variância (ANOVA), evidenciou diferença estatística com relação ao diâmetro longitudinal e transversal das plântulas, os dados foram coletados aos 30, 60, 90 e 120 dias, ao final do período observou-se que o tratamento com cama de frango seguida do esterco de gado estimularam os cladódios jovens propiciando maior diâmetro longitudinal e transversal tanto para a variedade de palma miúda quanto para a variedade orelha de elefante.

PALAVRAS-CHAVE: conservação, meio ambiente, biotecnologia.

\section{ACLIMATIZATION OF CACTUS PEAR GENOTYPES Opuntia stricta (Haw.) E Nopalea cochenilifera (L.) Salm-Dyck RESISTANT TO CARMINE COCHINEAL (Dactylopius opuntiae)}

\begin{abstract}
The irregularity of rain limits the natural production of animal feed in the Northeastern semi-arid region, it is used the forage palm as a strategic food resource, since this cactus has essential physiological characteristics, enabling economy and cultivation during a long period of drought. The aim of this work was to test how different types of substrate influence the growth of elephant ear and spineless cactus resistant to carmine mealybug during the acclimatization process. The Fragments of cladodes of two varieties of palm, spineless cactus and elephant ear, were grown in Murashige and Skoog (1962) culture medium, containing growth regulators, then the flasks were open, the seedlings were wash under running water
\end{abstract}

and subjected to acclimatization in a greenhouse with a system of programmed nebulization and constant temperature of $30^{\circ} \mathrm{C}$ in which four types of substrate were tested: clay sand, commercial organic substrate, clay sand with bovine manure and clay sand with poultry litter. After 120 days of monitoring, the analysis of variance test (ANOVA) showed a statistical difference in relation to the longitudinal and transversal diameter of the seedlings, the data were collected up to $30,60,90$ and 120 days, at the end of this period it was observed that the treatment of poultry litter followed by organic substrate stimulated young cladodes providing a greater longitudinal and 
transversal diameter for a spineless cactus variety and either an elephant ear variety.

KEYWORDS: conservation, environment, biotechnology. 


\section{INTRODUÇÃO}

A palma forrageira - Opuntia fícus-indica (L.) Mill. - cactácea exótica originária do México está presente em todos os continentes com diversas finalidades, destacando-se sua utilização na alimentação animal. Esta planta é um recurso alimentar estratégico para as regiões áridas e semiáridas do Nordeste brasileiro, já que é uma cultura que apresenta aspectos fisiológicos especiais, suportando prolongados períodos de estiagem (Neves et al., 2010).

Considerando a destruição dos palmais da variedade Gigante (Opuntia fícus indica Mill.) pela Cochonilha-do-carmim (Dactylopius opuntiae), e a procura por opção para abrandar as questões trazidas pela praga, foram introduzidas variedades resistentes ao inseto-praga, tais como a palma Orelha de Elefante Mexicana (Opuntia stricta Haw) e a Palma Miúda ou Doce (Nopalea cochenillifera Salm Dyck) (Araújo et al., 2019).

De acordo com a Organização das Nações Unidas (ONU), a palma está entre os alimentos prováveis na batalha contra a fome por exibir em sua composição alto valor nutricional, podendo se tornar uma alternativa viável para alimentação humana e animal, sendo utilizada em medicamentos contra gastrite, hiperglicemia, diabetes, arteriosclerose e hipertrofia prostática (Cândido Filho, Pereira \& Lima, 2014; Díaz, Rosa, Héliès-Toussaint, Guéraud \& Nègre-Salvayre, 2017).

A cochonilha-do-carmim (Dactylopius opuntiae) é considerada a principal praga da palma no Nordeste brasileiro (Almeida, Silva, Araújo, Oliveira \& Leite, 2011), devastando milhares de hectares em vários municípios, o que acarretou insegurança na oferta de alimento em período de estiagem.

Entre os aspectos essenciais para serem analisados no sistema de produção de mudas de boa qualidade, estão os substratos. Para Kämpf (2001), a seleção do substrato e o manejo são sérias questões técnicas para os viveiristas, pertinente à importância da obtenção de mudas de qualidade. Desse modo, emprego do substrato conveniente é um dos fatores essenciais para a produção de mudas que assegura o estabelecimento do plantio, restringe o tempo de formação e as perdas em campo (Vieira, Azevedo, Rodrigues \& Rossi, 1998).

As cactáceas são de extrema importância para a sustentabilidade do bioma Caatinga, servindo como fonte de forragem e alimento, especialmente nas épocas de seca prolongada, além de possuírem características de interesse medicinal e ornamental (Coelho, Fuck Júnior \& Nascimento, 2015).

As principais ameaças relatadas para a cactáceas estão relacionadas à fragmentação do hábitat, principalmente ocasionado pelo desmatamento, desenvolvimento agrícola e diversos tipos de distúrbios ambientais, como o trânsito de pessoas, expansão urbana e pisoteio por animais (Bárbara et al., 2015).

No decorrer dos últimos anos, foram desenvolvidas técnicas de cultivo in vitro para mais de mil espécies, abrangendo as cactáceas, como as Opuntias se multiplicam por estacas de cladódios, demandando grandes áreas para cultivo, a técnica de cultura de tecidos com o intuito de induzir brotos em laboratório e aclimatizar os mesmos em pequenos espaços (Villalobos, 2001). 
O esfagno é um musgo que cresce em regiões denominadas turfas, sendo que esta cobre somente 3\% da superfície terrestre (Charman, 2002). Dentre os substratos mais utilizados por viveiristas na aclimatização de plântulas como orquídeas está o esfagno, não se tem relato na literatura de pré-aclimatização de cactáceas com este material.

A aclimatização, em linhas gerais, consiste na transição do ambiente em que a planta se encontra em condições heterotróficas (in vitro) para condições autotróficas (ex vitro). Definido como a adaptação climática de um organismo, especialmente uma planta, que é transferida para um novo ambiente, sendo todo esse processo realizado artificialmente (Guerra, Torres \& Teixeira, 1999).

Pesquisas têm sido realizadas visando detectar os principais gargalos durante o processo de aclimatização, um grande número de plantas micropropagadas não sobrevive quando são transferidas das condições in vitro para o ambiente externo, sendo os principais problemas relatados associados aos seguintes pontos: baixa capacidade fotossintética, desidratação, raízes não funcionais, doenças, manutenção da alta umidade relativa após transplante e solo adequado (Souza \& Junghans, 2006).

A etapa denominada aclimatização, pode ser considerada a fase final da micropropagação e sua importância é tal que pode significar a limitação de todo o processo de multiplicação in vitro de plantas, para solucionar este problema as forrageiras tem sido melhoradas através de técnicas de cultura de tecidos vegetais (Spangemberg, 2001).

É necessário difundir as necessidades de harmonizar cada vez mais as condições de cultivo in vitro a um sistema mais próximo do natural, a utilização de casas de vegetação com sistemas de nebulização programada são soluções baratas e criativas (Gonçalves, 1993).

Diante do exposto, esse trabalho teve como objetivo testar a aclimatização das palmas forrageiras orelha de elefante e miúda oriunda da micropropagação em estufa de nebulização, utilizando diferentes tipos de substrato.

\section{MATERIAL E MÉTODOS}

\subsection{Estabelecimento e multiplicação in vitro}

Os experimentos foram conduzidos no Laboratório de Biotecnologia de Conservação de Espécies Nativas (LABCEN) localizado na UFRN, Rio Grande do Norte, em parceria com o Laboratório de Biotecnologia da EMPARN, plantas de palma forrageira das variedades Orelha de elefante e miúda resistentes à cochonilha-do-carmim (Dactylopius opuntiae) foram estabelecidas (Figura 1A) e posteriormente multiplicadas in vitro em meio de cultura Murashige e Skoog (1962) suplementado com BAP (Figura 1B). As culturas foram mantidas em sala de crescimento com temperatura controlada $25 \pm 2$ ㅇ C, sob fotoperíodo de $16 / 8$ horas de luz/escuro e umidade relativa de $70 \%$, em delineamento experimental inteiramente casualizado (Figura $1 \mathrm{C}$ ). 


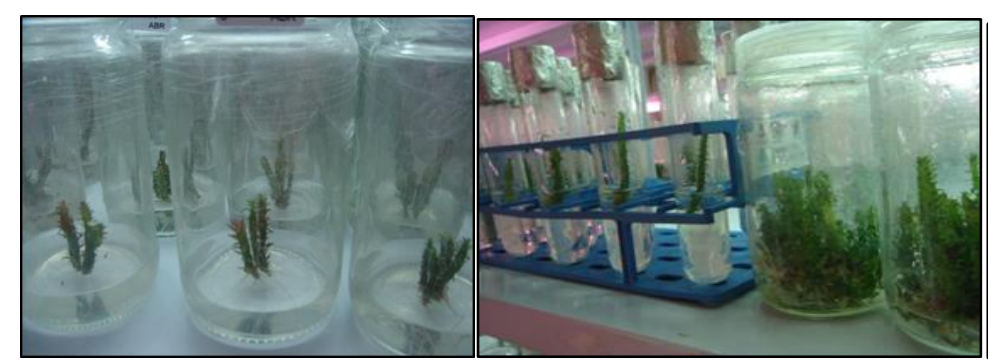

A

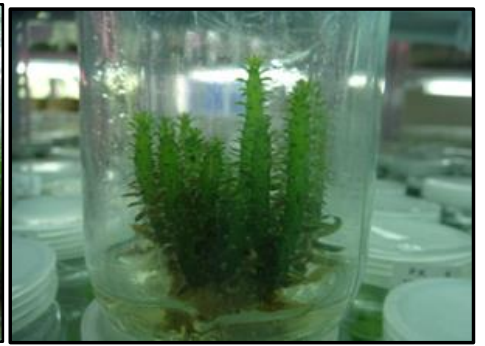

C

Figura 1: Estabelecimento da cultura (A); Fase de multiplicação in vitro (B); Ambiente controlado (C)

\subsection{Pré-Aclimatização}

As mudas foram retiradas dos frascos, individualizadas (Figura $2 \mathrm{~A}$ ) e lavadas em água corrente, sendo acomodadas em bandejas (Figura 2B), as mesmas foram postas sobre a bancada do laboratório para pré-aclimatização em esfagno durante 20 dias. As plantas foram pulverizas 3 (três) vezes ao dia com água utilizando borrifador manual.

As posições que as mudas foram colocadas no esfagno influenciaram significativamente no sucesso da aclimatização (Figura $2 \mathrm{C}$ ), pois observamos que as perdas por apodrecimento foram minimizadas com espaçamento de aproximadamente 2 (dois) $\mathrm{cm}$ entre as mudas.

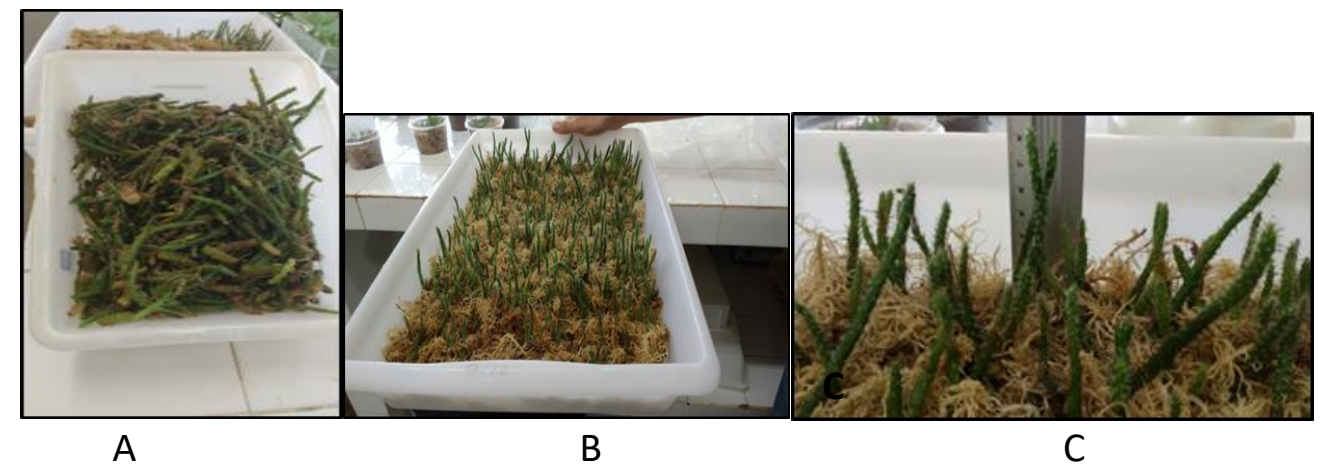

Figura 2: Mudas lavadas resultantes da fase de micropropagação (A); Etapa de pré-aclimatização em esfagno (B); detalhe do espaçamento entre as mudas pré-aclimatizadas (C).

\subsection{Aclimatização}

Após 20 dias no esfagno, as plantas das duas variedades de palma foram transferidas para ambiente protegido, com irrigação programada e nebulização 3 (três) vezes por dia por 5 minutos, onde foram testados 4 tipos de substrato: Areia, Areia + adubo orgânico comercial (POLE) (1:1), Areia + esterco de gado (1:1) e Areia + cama de frango(1:1). Com o auxílio de um paquímetro universal, foi realizada a mensuração do diâmetro longitudinal e diâmetro transversal dos cladódios (Figura 3), medidas feitas de 30 em 30 dias até atingir 120 dias em estufa de nebulização (Figura 4). Foram instaladas duas áreas experimentais para validar o processo de micropropagação, plantas aclimatizadas foram implantadas em campo sob condições naturais, foram escolhidas uma área na EMPARN e outra na UFRN (Figura 5 A e B). 


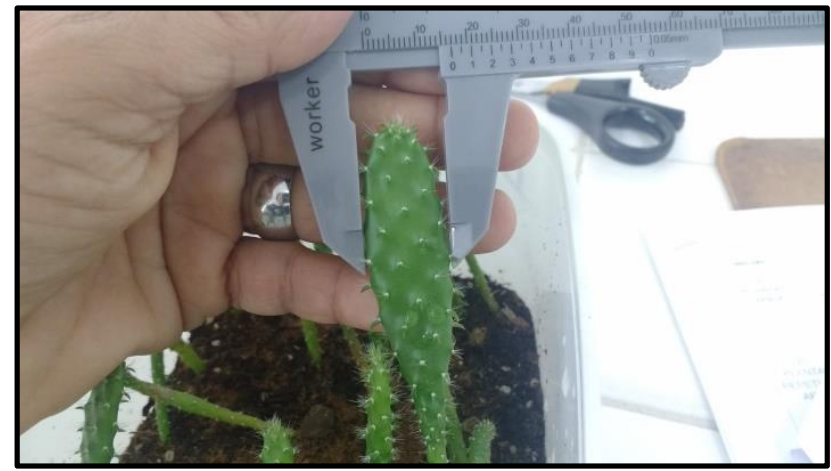

Figura 3: Uso do paquímetro universal para efetuar as mensurações de diâmetro longitudinal e diâmetro transversal.

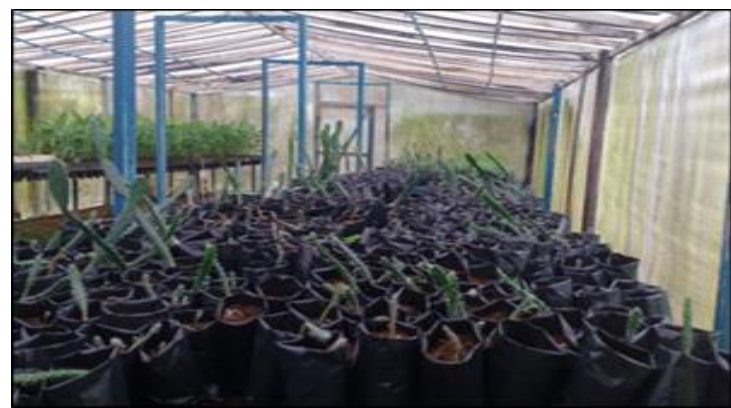

Figura 4: Plantas individualizadas em aclimatização sob nebulização programada

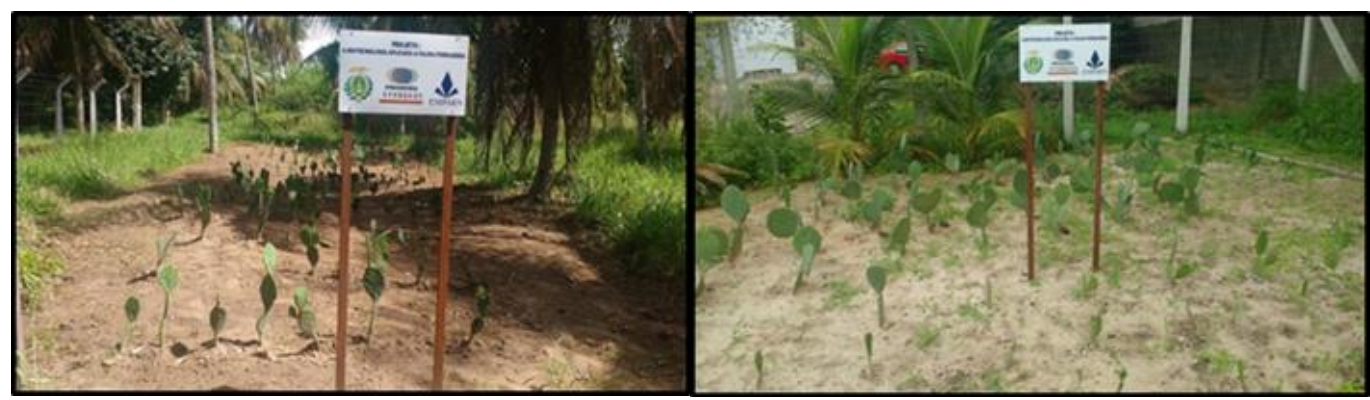

Figura 5: Material plantado no campo em condições naturais, implantadas em duas áreas experimentais localizadas na EMPARN (A) e UFRN (B)

\subsection{ANÁLISE DE MACRO E MICRONUTRIENTES DOS CLADÓDIOS APÓS ACLIMATIZAÇÃO}

Após 120 dias em ambiente protegido, as plantas sobreviventes das duas variedades foram retiradas dos substratos, lavadas, identificadas, agrupadas de acordo com o substrato testado e a variedade e fotografadas, em seguida foram enviadas para o Laboratório de análises de solo, água e planta da EMPARN para serem analisadas quanto aos macro e micronutrientes.

\subsection{ANÁLISE ESTATÍSTICA}

O delineamento experimental foi o de blocos inteiramente casualizados com 4 (quatro) tratamentos e vinte repetições. Para testar como diferentes tratamentos influenciam no crescimento das palmas Orelha de elefante e Miúda, fizemos dois modelos de ANOVA de medidas 
repetidas utilizando a função "aov" do software R. No primeiro modelo, a variável resposta utilizada foi a medida longitudinal de crescimento das duas espécies durante 120 dias e no segundo modelo a variável resposta utilizada foi a medida transversal de crescimento das duas espécies durante 120 dias. Para ambos os modelos utilizamos como variáveis predetoras os tipos de substrato: Areia, Areia+ adubo, Areia + esterco e cama de frango e a espécie de palma (Becker, 2015; Casella \& Berger, 2010).

\section{RESULTADOS E DISCUSSÃO}

$\mathrm{Na}$ etapa de estabelecimento in vitro, foi observada a contaminação por fungos e bactérias nas culturas em torno de 10\%, fato este validado por Xavier, Wendling e Silva (2009), que relataram a contaminação de explantes no cultivo in vitro devido às condições naturais que as plantas se encontram, ricas em microorganismos. Os reguladores de crescimento determinaram o desenvolvimento e induziram brotações nas variedades de palma orelha de elefante e miúda, segundo Rocha (2009) as mudas submetidas a reguladores de crescimento devem apresentar capacidade de responder a estímulos específicos, e estes resultados foram confirmados pela quantidade de mudas geradas, chegando a obter-se em torno de 20 brotos por explante inoculado, havendo um crescimento significativo em cada repicagem com o intervalo de 20 dias entre uma avaliação e outra.

A pré-aclimatização em esfagno foi determinante para a sobrevivência das mudas, pois a aclimatização é uma etapa crítica no processo de cultivo in vitro, e pode comprometer a produção de mudas de algumas espécies, considerando que as plantas são expostas a mudanças bruscas no que diz respeito às condições ambientais, sendo a perda de água um dos principais problemas (Souza, Silva \& Araújo, 2015). A sobrevivência nesta etapa foi de $100 \%$, possibilitando um número significativo de plantas viáveis, Silva e Ferreira (2016) confirmam a obtenção de explantes em diversos ciclos em cactáceas. Observamos que devemos sempre preservar a distância entre as plantas para não induzir o aumento da umidade na bandeja e apodrecer o material, as qualidades básicas e indispensáveis de um substrato são: consistência para suporte, boa aeração das raízes, capacidade de retenção de água, alta durabilidade e pH adequado (Silva \& Silva, 1997; Kämpf, 2000; Silva, 2000; Souza, 2003).

As duas variedades orelha de elefante e miúda apresentaram um desenvolvimento de cladódios inferior aos demais no substrato areia, este atuou como grupo controle (Figura 6 A e B), onde pudemos observar uma quantidade de raízes nas plantas superior aos demais substratos testados. É essencial para a sobrevivência da planta, que a parte aérea seja bem desenvolvida e de aspecto sadio, já que a atividade radicular é inicialmente bastante reduzida (Souza \& Junghans, 2006). A principal vantagem do enraizamento ex vitro é o menor custo que esta técnica proporciona, um dos grandes gargalos da cultura de tecidos de plantas são os custos com a produção segundo Souza, Silva \& Araújo (2015). 


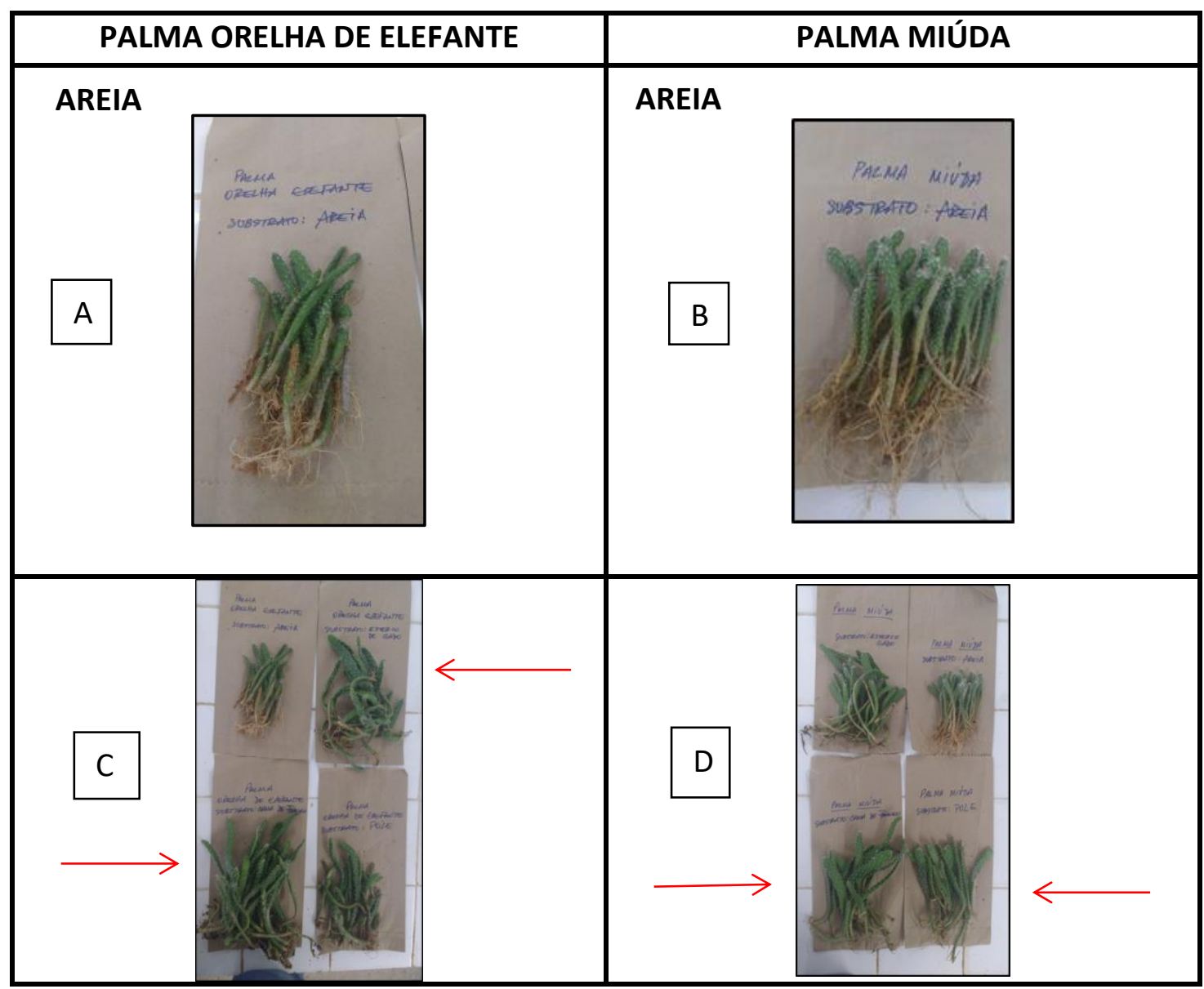

Figura 6: cladódios agrupados de acordo com as variedades e substrato testados

Durante o crescimento em estufa de nebulização (aclimatização), foi observada a taxa de sobrevivência de 100\%, após 120 dias para ambas as variedades (Figura 6C e D); na figura 6C está registrada a variedade Orelha de elefante em fotos comparativas, onde podemos observar que no substrato "Areia+cama de frango" e "Areia+esterco de gado" as plantas alcançaram melhor desenvolvimento. $\mathrm{Na}$ figura 6D podemos observar que na variedade Miúda houve melhor desenvolvimento no substrato "Areia + Cama de frango e Areia + adubo orgânico.

Pudemos observar que os cladódios que foram pré-aclimatizadas ao serem submetidas a temperaturas mais altas na estufa de nebulização sofreram inicialmente perda de água, apresentando murchamento, porém recuperaram sua vitalidade $100 \%$ em aproximadamente 5 dias sob nebulização automatizada. Segundo Faria, Assis \& Carvalho (2010), o substrato utilizado para aclimatização deve apresentar características satisfatórias quanto à economia hídrica, aeração, permeabilidade, poder de tamponamento para valores de $\mathrm{pH}$ e capacidade para retenção de nutrientes.

Quanto à análise dos macronutrientes e micronutrientes nos cladódios jovens após 120 dias, foram obtidos valores em destaque para os substratos "areia + cama de frango" comuns às duas 
variedades de palma, o diagnóstico da parte aérea das plantas, contribui no acompanhamento das necessidades de diversas culturas durante o crescimento, as análises foliares nos orientam a ter uma melhor previsão sobre o estado nutricional e as deficiências das plantas analisadas salientando onde são essenciais ajustes dentro do esquema de adubação ou escolha do substrato adequado. Segundo Malavolta (1980), quando sintomas visuais não são claros ou não são visíveis, a análise dos macro e micronutrientes da parte aérea deve ser usada para diagnosticar ou descartar deficiências nutricionais.

\begin{tabular}{|c|c|c|c|c|c|c|c|c|c|c|}
\hline Substratos & \multicolumn{5}{|c|}{ Macronutrientes g. $\mathrm{kg}^{-1}$} & \multicolumn{5}{|c|}{ Micronutrientes $\mathrm{mg} \cdot \mathrm{Kg}^{-1}$} \\
\hline & $\mathbf{N}$ & $\mathbf{P}$ & K & $\mathrm{Ca}$ & $\mathrm{Mg}$ & $\mathrm{Na}$ & $\mathrm{Zn}$ & $\mathrm{Cu}$ & $\mathrm{Fe}$ & $\mathrm{Mn}$ \\
\hline Areia & 14,01 & 1,40 & 28,52 & 10,97 & 9,37 & 8,85 & 85 & 15 & 2,575 & 90 \\
\hline *Areia+adubo & 15,97 & 7,51 & 17,36 & 12,98 & 19,00 & 4,13 & 77 & 16 & 5,812 & 91 \\
\hline Areia+esterco & 17,93 & 6,61 & 47,12 & 14,36 & 17,62 & 11,80 & 73 & 13 & 727 & 114 \\
\hline $\begin{array}{c}\text { Areia+cama } \\
\text { de frango }\end{array}$ & 20,17 & 9,88 & 22,32 & 35,50 & 29,75 & 5,31 & 100 & 18 & 716 & 67 \\
\hline
\end{tabular}

*Foi utilizado o adubo orgânico POLE fértil

Quadro 1: Análise dos cladódios da PALMA ORELHA DE ELEFANTE

\begin{tabular}{|c|c|c|c|c|c|c|c|c|c|c|}
\hline Substratos & \multicolumn{4}{|c|}{ Macronutrientes g.kg ${ }^{-1}$} & \multicolumn{5}{c|}{ Micronutrientes $\mathrm{mg} \mathrm{Kg}^{-1}$} \\
\hline & $\mathrm{N}$ & $\mathrm{P}$ & $\mathrm{K}$ & $\mathrm{Ca}$ & $\mathrm{Mg}$ & $\mathrm{Na}$ & $\mathrm{Zn}$ & $\mathrm{Cu}$ & $\mathrm{Fe}$ & $\mathrm{Mn}$ \\
\hline Areia & 17,93 & 1,65 & 22,32 & 6,77 & 6,22 & 8,26 & 88 & 23 & 2.587 & 91 \\
\hline *Areia+adubo & 16,25 & 6,97 & 22,32 & 25,50 & 31,12 & 5,31 & 91 & 18 & 1.300 & 122 \\
\hline Areia+esterco & 17,93 & 3,29 & 26,04 & 14,95 & 17,12 & 7,08 & 64 & 11 & 950 & 142 \\
\hline $\begin{array}{c}\text { Areia+cama de } \\
\text { frango }\end{array}$ & 18,21 & 7,83 & 18,97 & 37,82 & 27,25 & 4,72 & 100 & 14 & 950 & 67 \\
\hline
\end{tabular}

*Foi utilizado o adubo orgânico POLE fértil

\section{Quadro 2: Análise dos cladódios da PALMA MIÚDA}

Observamos que os valores do macronutriente Nitrogênio $(N)$ encontra-se superior nas plantas que foram cultivadas em areia + cama de frango e areia + esterco de gado para a variedade orelha de elefante (Quadro 1), e para a variedade miúda temos como destaque areia + cama de frango e areia + adubo orgânico comercial (Quadro 2) esta análise confirmou o que foi observado nas fotos comparativas de crescimento. O nitrogênio atua na forma estrutural da planta, fazendo parte da molécula dos compostos orgânicos do carbono, tais como os diversos aminoácidos formadores das proteínas, enzimas e coenzimas, além de ser constituinte das moléculas de clorofila (Malavolta, 1980), sendo de extrema importância para a formação de parte aérea das plantas e consequentemente a pega da muda no campo.

Segundo Silva e Ferreira (2016), cladódios mais desenvolvidos podem favorecer a sobrevivência das plantas durante o processo de aclimatização, bem como propiciar a obtenção de um número superior de plantas, caso a planta seja fracionada para a obtenção de explantes em novos ciclos de multiplicação in vitro, este fato foi comprovado pelos nossos experimentos durante as etapas de desenvolvimento com as duas variedades de palma Figura 7 A e B. 


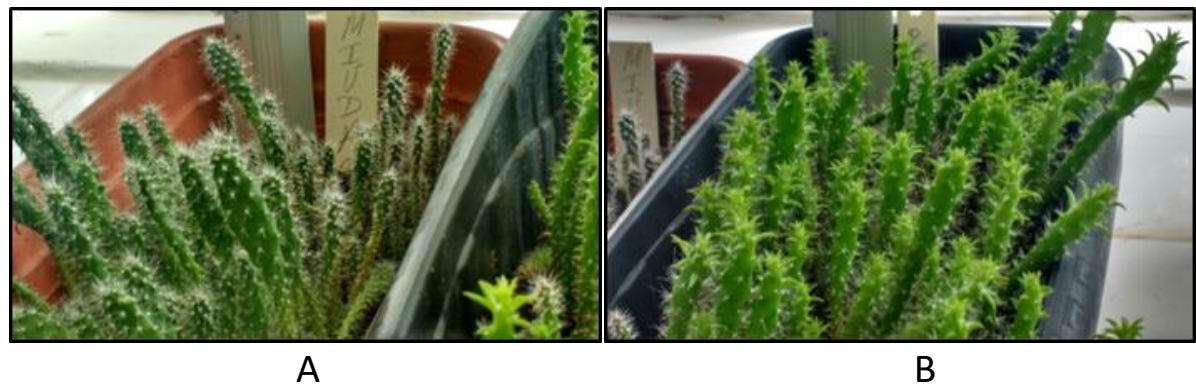

Figura 7: Palmas em fase final de aclimatização miúda (A) e orelha de elefante (B)

\section{DIÂMETRO LONGITUDINAL}

As espécies não diferiram em crescimento do diâmetro longitudinal ao longo do tempo, mas tiveram diferenças entre os tratamentos (Tabela 1, Figura 8). Os tratamentos de areia + adubo, areia + esterco e cama de frango apresentaram maiores crescimento aos tratamentos de areia pura. 0 tratamento cama de frango apresentou maior crescimento em relação ao tratamento areia+adubo (Tabela 2). O maior fornecimento de matéria orgânica ao solo promove melhorias aos atributos físicos como os diâmetros dos cladódios, em função de possibilitar melhores condições ao desenvolvimento das plantas, ocorrendo um maior desempenho físico e nutricional (Borba, Silva \& Andrade, 2008).

Tabela 1: Tabela de ANOVA testando o efeito de diferentes substratos e das espécies de palma "orelha de elefante" e "Miúda"

\begin{tabular}{llllll}
\hline \multicolumn{2}{l}{ Diâmetro Longitudinal } \\
\hline Erro dias & $\mathrm{GL}$ & $\mathrm{SQ}$ & & & \\
Residuos & 1 & 3050 & & & \\
\hline Erro interno & $\mathrm{GL}$ & $\mathrm{SQ}$ & $\mathrm{MQ}$ & F-valor & p-valor \\
\hline Tratamentos & $\mathbf{3}$ & $\mathbf{2 0 4 8}$ & $\mathbf{6 8 2 . 5}$ & $\mathbf{5 2 . 9 9 3}$ & $<\mathbf{0 . 0 0 1}$ \\
Espécie & 1 & 21 & 20.6 & 1.599 & 0.207 \\
Residuos & 634 & 8166 & 12.9 & & \\
\hline
\end{tabular}

Tabela 2: Análises pos-hoc identificando quais tratamentos diferem entre si.

\begin{tabular}{lllll}
\hline Crescimento longitudinal - Pos Hoc & & & & \\
\hline & diferença & limite inferior & & limite superior \\
p-valor
\end{tabular}




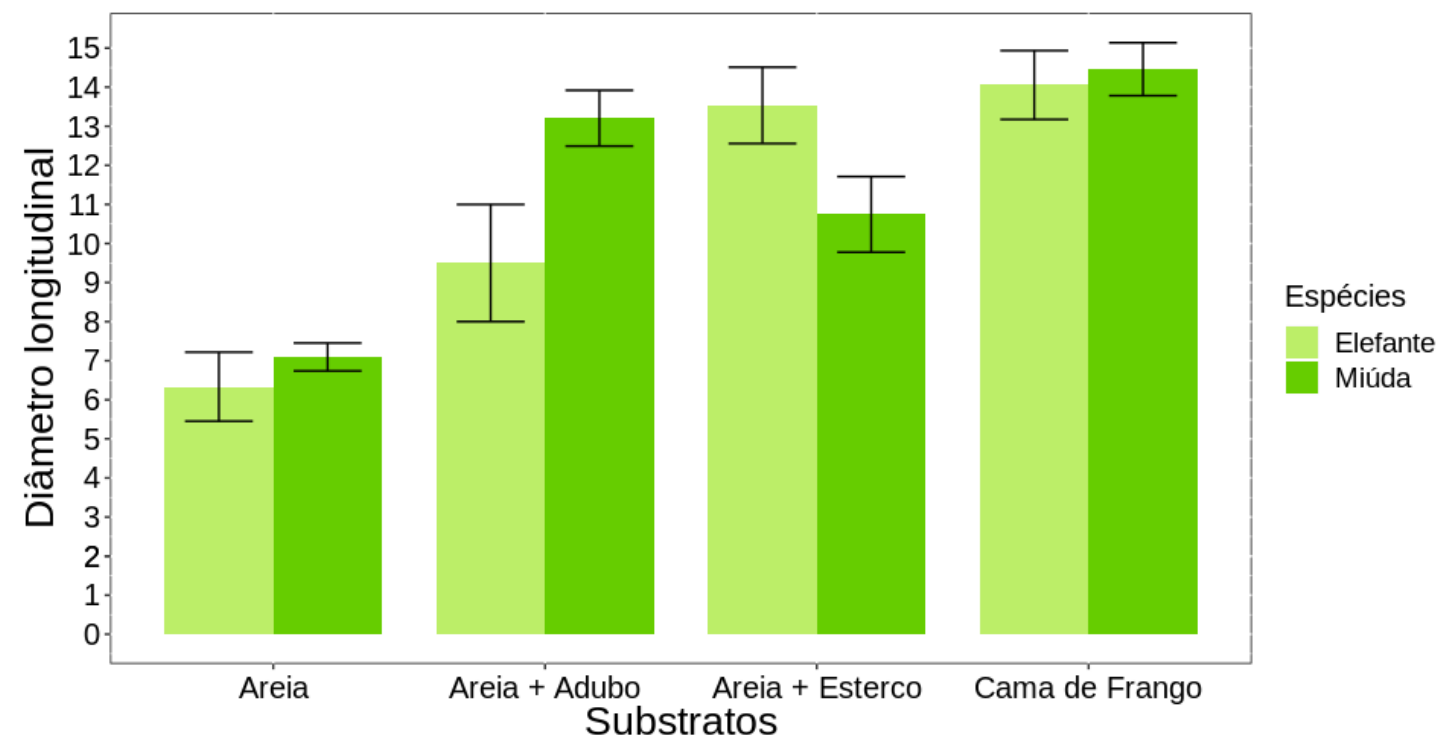

Figura 8: Crescimento longitudinal em 120 dias das palmas "Elefante" e "Miúda" em diferentes tipos de substratos.

Resultados semelhantes aos nossos foram obtidos por Silva et al. (2018), que trabalhando com adubação orgânica em cactáceas, observaram que os maiores incrementos nos atributos físicos foram obtidos nas plantas que receberam a adubação bovina, para os diâmetros longitudinal e transversal de cladódios, respectivamente.

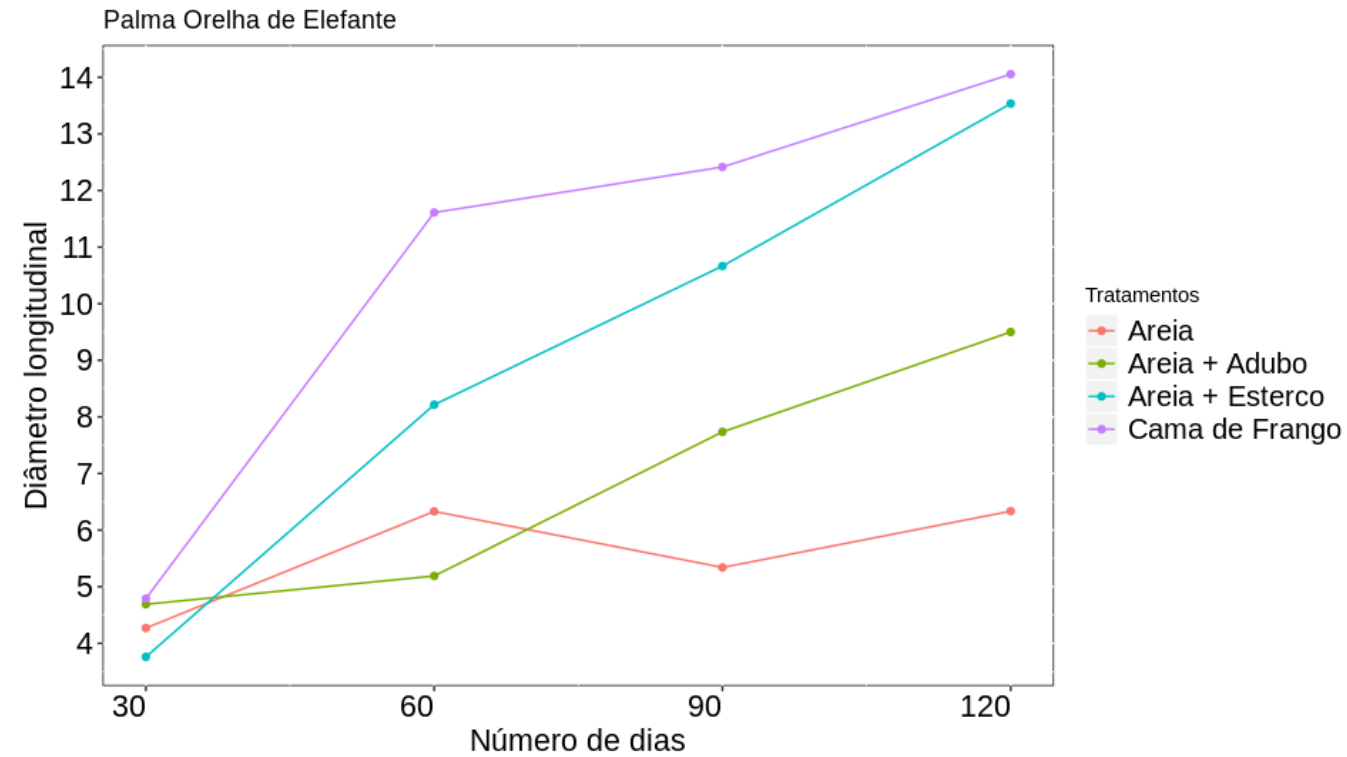

Figura 9: Crescimento longitudinal ao longo do tempo da palma "Orelha de elefante" em diferentes tratamentos de substrato. 


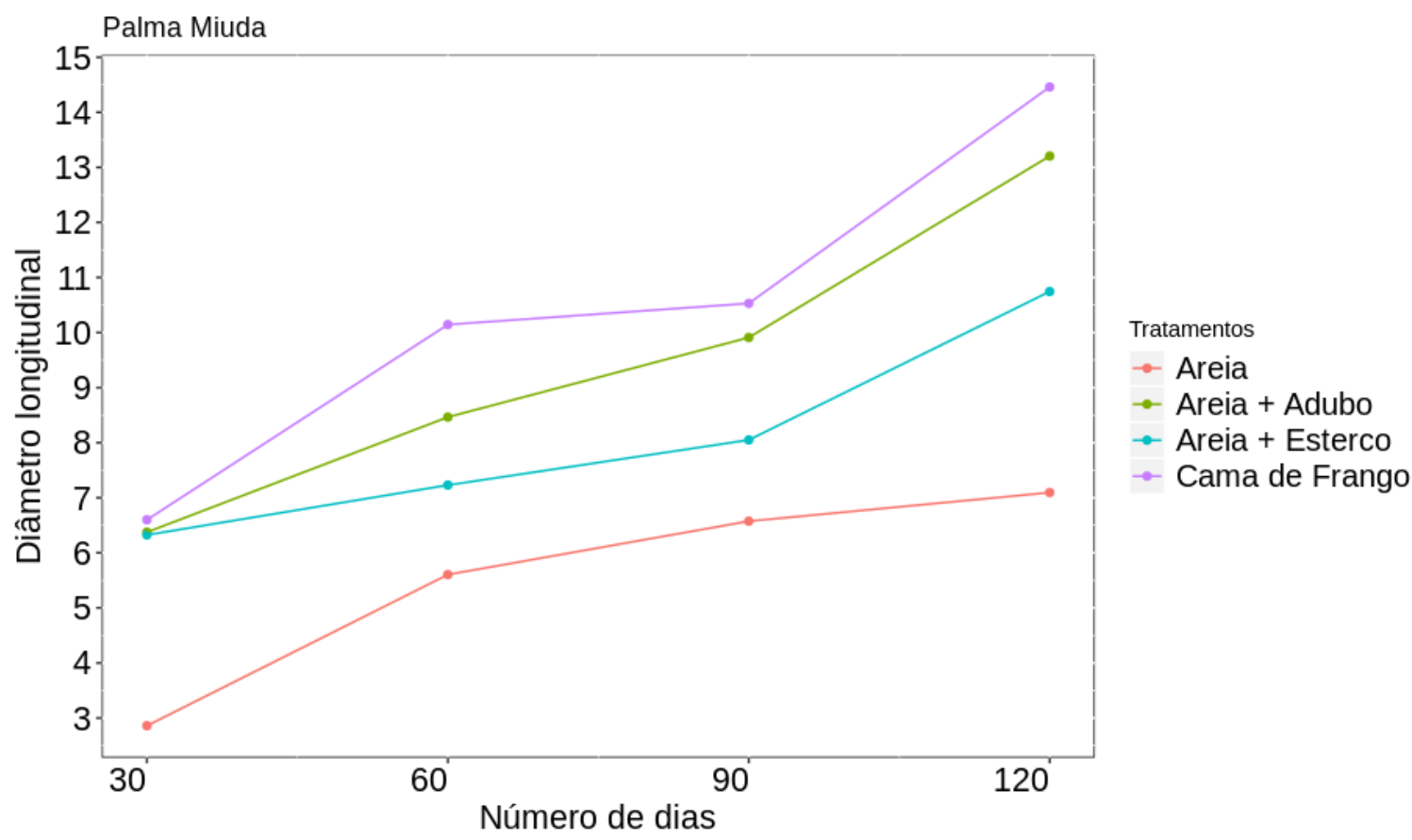

Figura 10: Crescimento longitudinal ao longo do tempo da palma "Miúda" em diferentes tratamentos de substrato.

\section{DIÂMETRO TRANSVERSAL}

O diâmetro transversal diferiu tanto entre tratamentos, quanto entre as espécies de palma (Tabela 3, Figura 11). De forma geral, a palma "Miúda" apresentou maior crescimento transversal do que a palma "Orelha de elefante". Assim como para o crescimento longitudinal os tratamentos Areia + adubo, Areia + esterco e Cama de Frango apresentaram maiores crescimentos do que o tratamento Areia. O tratamento cama de frango apresentou crescimento transversal superior ao tratamento Areia + esterco (Tabela 4).

Tabela 3: Tabela de ANOVA testando o efeito de diferentes substratos e das espécies de palma "orelha de elefante" e "Miúda".

\begin{tabular}{lllll}
\hline \multicolumn{5}{l}{ Diâmetro Transversal } \\
\hline Erro dias & $\mathrm{GL}$ & $\mathrm{SQ}$ & & \\
Residuals & 1 & 9.569 & & \\
\hline Erro interno & $\mathrm{GL}$ & $\mathrm{SQ}$ & F-valor & p-valor \\
\hline Tratamento & 3 & 26.44 & 41.447 & $<0.001$ \\
Espécie & 1 & 0.83 & 3.907 & 0.048 \\
Resíduos & 634 & 134.81 & & \\
\hline
\end{tabular}

Tabela 4: Análises pos-hoc identificando quais tratamentos diferem entre si.

\section{Crescimento transversal}

\begin{tabular}{lllll}
\hline & diferença & limite infeior & limite superior & $\mathrm{p}$-valor \\
\hline Areia + adubo X Areia & 0.1927878 & 0.0638543 & 0.3217214 & 0.0008688
\end{tabular}




$\begin{array}{lllll}\text { Areia + Esterco x Areia } & 0.1791459 & 0.0502124 & 0.3080795 & 0.0023242 \\ \text { Cama de Frango x Areia } & 0.3113663 & 0.182432 & 0.4402999 & <0.001 \\ \text { Areia + esterco X Areia + Adubo } & -0.013641 & -0.1425754 & 0.1152916 & 0.9927238 \\ \text { Cama de frango X Areia + Adubo } & 0.1185785 & -0.0103549 & 0.2475121 & 0.0835246 \\ \text { Cama de Frango x Areia + Esterco } & 0.1322204 & 0.0032869 & 0.261154 & 0.0420998\end{array}$

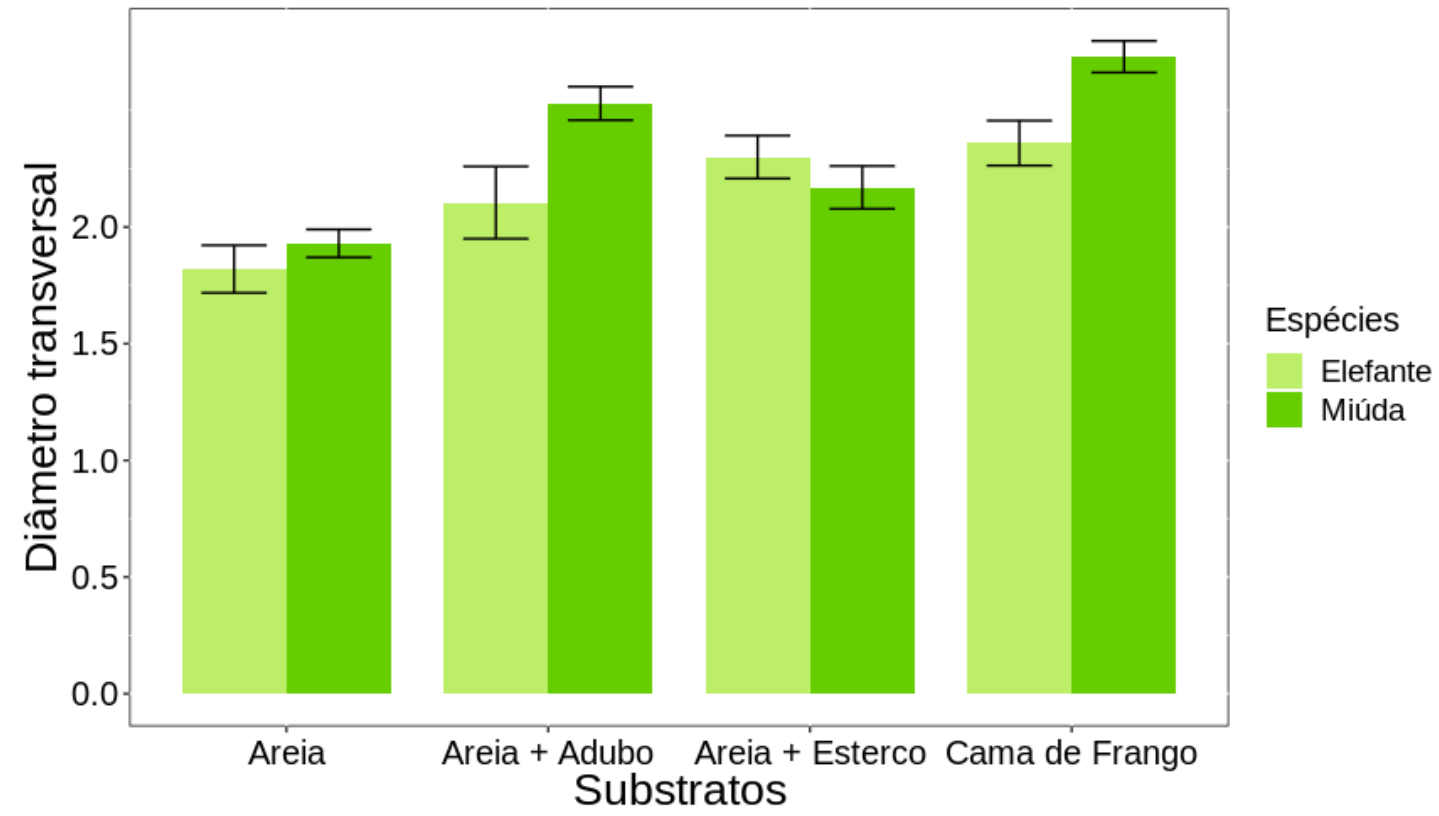

Figura 11: Crescimento transversal em 120 dias das palmas "Elefante" e "Miúda" em diferentes tipos de substratos.

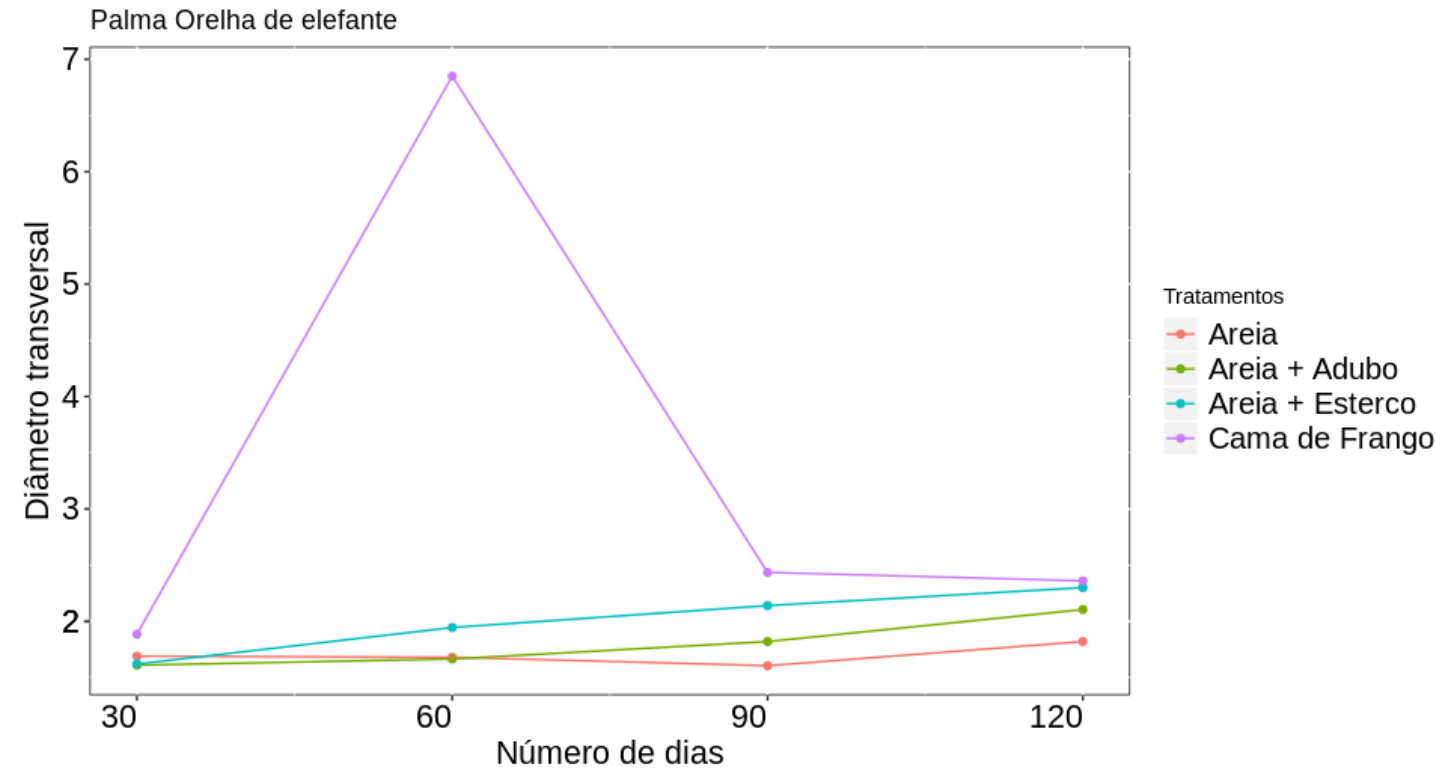

Figura 12: Crescimento transversal ao longo do tempo da palma "Orelha de elefante" em diferentes tratamentos de substrato. 


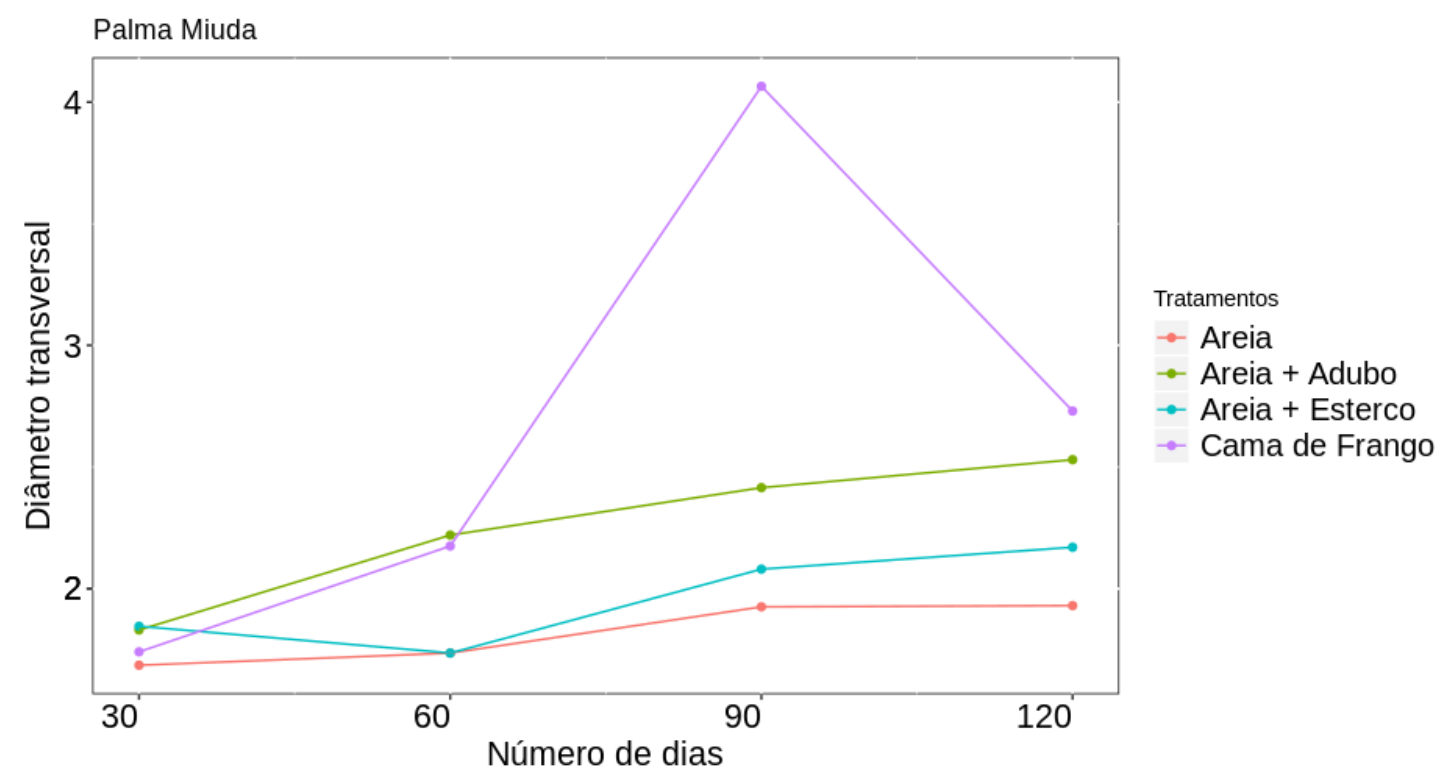

Figura 13: Crescimento transversal ao longo do tempo da palma "Miúda” em diferentes tratamentos de substrato.

Um maior fornecimento de matéria orgânica ao solo possibilita um maior balanceamento nutricional e fisiológico, e consequentemente, favorece a produção e acúmulo de fotoassimilados (Souza et al., 2018), o que foi confirmado no nosso experimento com o maior desenvolvimentos dos cladódios em estufa para as duas variedades de palma, pois a cama de frango promove a absorção da agua dos bebedouros e ainda recebe restos de rações e penas (Correa \& Miele, 2011).

De acordo com Correa e Miele (2011) a adubação com cama de aves quando efetuada de maneira adequada promove um acréscimo no potencial de produção agrícola. Entre os nutrientes presentes na cama de frango o nitrogênio está em maior concentração, devido à dieta das aves o que foi confirmado na análise dos macronutrientes dos cladódios das duas variedades em nosso experimento (Aita et al., 2013).

Observamos durante os experimentos que a escolha dos recipientes e substratos foi de extrema importância para a saúde e preservação das mudas na pré-aclimatização e aclimatização, evitando que morressem por apodrecimento ou pouca drenagem, segundo Silva (2017) o tipo de recipiente (bandejas, tubetes, sacos plásticos ou copos descartáveis) é de extrema importância para o sucesso da aclimatização.

\section{CONCLUSÕES}

Estabelecimento e micropropagação de palma forrageira variedades miúda e orelha de elefante são viáveis em meio MS, 1962 suplementado com BAP;

O esfagno pode ser usado como substrato na fase de pré-aclimatização para a cultura de palma forrageira;

Mudas de palma forrageira orelha de elefante e miúda multiplicadas em laboratório podem ser utilizadas para propagação de novos genótipos tolerantes a cochonilha-do-carmim, comparadas 
ao método tradicional, desde que em condições controladas de propagação e aclimatização em estufa de nebulização;

Os cladódios de comprimento superior apresentaram maiores atributos físico e físicoquímicos na palma "Orelha de Elefante Mexicana" sendo indicada para o plantio;

A aclimatização com substrato "cama de frango" se destacou propiciando maior desenvolvimento de cladódios jovens com diâmetro longitudinal e transversal superiores, tanto para a variedade de palma miúda quanto para a variedade orelha de elefante;

A Palma forrageira é uma planta geradora de renda, que viabiliza a permanência do homem no campo, a técnica de micro propagação pode ser utilizada como estratégia de recuperação de áreas degradadas e colaborar na restauração da caatinga;

A taxa de sobrevivência foi de $100 \%$ para as duas variedades de palma forrageira, comprovando a viabilidade técnica.

\section{REFERÊNCIAS}

Aita, C., Balem, A., Pujol, S. B., Schirmann, J., Gonzatto, R., Giacomini, D. A., Vargas, P. V. \& Giacomini, S. J. (2013). Redução na velocidade da nitrificação no solo após aplicação de cama de aviário com dicianodiamida. Ciência Rural, 43(8), 1387-1392. Recuperado de https://doi.org/10.1590/S0103-84782013005000102.

Almeida, A. A., Silva, R. A., Araújo, W. L., Oliveira, A. V. B. \& Leite, D. T. (2011). Problemas fitossanitários causados pela Cochonilha-do-Carmim a palma forrageira no Cariri Ocidental Paraibano. Revista Verde de Agroecologia e Desenvolvimento Sustentável, 6(3), 98-108. Recuperado de https://www.gvaa.com.br/revista/index.php/RVADS/article/view/743/671.

Araújo, J. S., Pereira, D. D., Lira, E. C., Félix, E. S., Souza, J. T. A. \& Lima, W. B. (2019). Palma forrageira: plantio e manejo. Campina Grande/PB: INSA. Recuperado de https://portal.insa.gov.br/images/acervo-livros/Cultivo\%20palma_final\%20gr\%C3\%A1fica.pdf.

Bárbara, E. P.; Silva, A. A.; Souza, M. M.; Gurgel, Z. E.; Marchi, M. N. \& Bellintani, M. C. (2015). Germinação e Criopreservação de Sementes de Cactos Nativos da Bahia. Gaia Scientia, 9(2), 9196. Recuperado de https://periodicos.ufpb.br/index.php/gaia/article/view/24315/13336.

Becker, J. L (2015). Estatística Básica: transformando dados em informação. Porto Alegre: Editora Bookman.

Borba, M. A. P.; Silva, D. S. \& Andrade, A. P (2008). A palma no Nordeste e seu uso na alimentação animal. Anais do Congresso Nordestino de Produção Animal, Simpósio Nordestino de Alimentação de Ruminantes, Simpósio Sergipano de Produção Animal, Aracaju, Brasil, 5, 11, 1. Recuperado de http://www.alice.cnptia.embrapa.br/handle/doc/894675.

Cândido Filho, A., Pereira, F. C. \& Lima, A. K. V. O. (2014). Base alimentar humana com o uso da palma forrageira: o estudo de arte. Anais do Simpósio de Reforma Agrária e Questões Rurais, 
Recife, Brasil, $6 . \quad$ Recuperado de https://www.uniara.com.br/legado/nupedor/nupedor_2014/Arquivos/03/6_Amy\%20Lima.pd f.

Casella, G. \& Berger, R. L. (2010). Inferência Estatística. São Paulo: Cengage Learning.

Charman, D. J. (2002). Peatlands and Environmental Change. New York: J. Wiley.

Coelho, Paulo J. A., Fuck Júnior, Sérgio C. F. \& Nascimento, Evaldo (2015). Coleta e conservação ex situ de cactáceas nativas do estado do Ceará. Gaia Scientia, 9(2) 183-192. Recuperado de https://periodicos.ufpb.br/index.php/gaia/article/view/27227/14548.

Correa, J. C. \& Miele, M. (2011). A cama de aves e os aspectos agronômicos, ambientais e econômicos. In Palhares, J. C. P. \& Kunz, A. Manejo ambiental na avicultura. Concórdia: Embrapa Suínos e Aves. (Embrapa Suínos e Aves. Documentos, 149). Recuperado de https://www.embrapa.br/suinos-e-aves/busca-de-publicacoes/-/publicacao/920818/a-camade-aves-e-os-aspectos-agronomicos-ambientais-e-economicos.

Díaz, M. S. S., Rosa, A. P. B., Héliès-Toussaint, C., Guéraud, F. \& Nègre-Salvayre, A. (2017). Opuntia spp.: Characterization and Benefits in Chronic Diseases. Oxidative Medicine and Cellular Longevity. 1-17. Recuperado de doi:10.1155/2017/8634249.

Faria, R. T., Assis, A. M. \& Carvalho, J. F. R. P. (2010). Cultivo de orquídeas. Londrina: Mecenas.

Gonçalves, A. C.R (1993). Aclimatização de plântulas. ABCTP Notícias, Brasília/DF, (20), 8.

Guerra, M. P., Torres, A. C. \& Teixeira, J. B. (1999). Embriogenese somática e sementes sintéticas. In Torres, A. C., Caldas, L. S. \& Buso, J. A. Cultura de tecidos e transformações genética de plantas (v.2). Brasília: Embrapa-CNPH.

Kämpf, A. N. (2000). Produção comercial de plantas ornamentais. Guaíba: Agropecuária.

Kämpf, A. N. (2001). Análise física de substratos para plantas (Boletim Informativo, v. 26). Viçosa: SBCS.

Malavolta, E. (1980). Elementos de nutrição mineral das plantas. São Paulo: Agronômica Ceres.

Murashige, T. \& Skoog, F. (1962). A revised medium for rapid growth and bioassays with tobacco tissue culture. Physiologia Plantarum. 15, 473-497.

Neves, A. L. A., Pereira, L. G. R., Santos, R. D., Voltolini, T. V., Araújo, G. G. L. de, Moraes, S. A. de, Aragão, A. S. L. \& Costa, C. T. F. (2010). Plantio e uso da palma forrageira na alimentação de bovinos no semiárido brasileiro (Embrapa Gado de Leite. Comunicado Técnico, 62). Juiz de Fora: Embrapa Gado de Leite. Recuperado de http://ainfo.cnptia.embrapa.br/digital/bitstream/item/42960/1/Salete-Meta-2011.pdf. 
Rocha, H. S (2009). Biofábricas: estrutura física e organização. In Junghans, T. G. \& Souza, A. da S. Aspectos práticos da micropropagação de plantas. Brasília/DF: Embrapa.

Silva, F. S. C. \& Silva, S. P. C. (1997). O substrato na cultura das orquídeas, sua importância, seu envelhecimento. Revista Oficial do Orquidário, 11 (1), 3-10.

Silva, F. S. C. (2000). Haverá algum substrato que substitua o xaxim? Boletim CAOB, Rio de Janeiro, $1(44), 68-76$.

Silva, M. M. A. \& Ferreira, L. T. (2016). Cultivo in vitro de plantas e suas aplicações em cactáceas. Campina Grande: Instituto Nacional do Semi Árido.

Silva, M. M. A. (2017). Micropropagação da palma forrageira variedade Miúda em meio de cultura simplificado. Tecnologia \& Ciência Agropecuária, 11 (2), 25-29. Recuperado de https://revistatca.pb.gov.br/edicoes/volume-11-2017/v-11-n-2-junho-2017/tca11205.pdf.

Silva, M. S., Costa, F. B., Formiga, A. S., Nóbrega, J. S., Brasil, Y. L., Lira, R. P., Silva, J. L., Silva, K. G., Sales, G. N. B., Pereira, U. S., Nascimento, A. M.(2018). Qualitative assessment of early palm cladodes of Opuntia tuna L. (Mill) grown with organic manures. Journal of Experimental Agriculture International, 26 (6), 1-14. Recuperado de https://www.journaljeai.com/index.php/JEAl/article/view/27419/51445.

Souza, A. S. \& Junghans, T. G. (2006). Introdução à micropropagação de plantas. Cruz das Almas: Embrapa Mandioca e Fruticultura Tropical.

Souza, F. M., Pereira, W. E., Dantas, J. S., Nóbrega, J. S., Lima, E. C. S. \& Sá, F. V. S. (2018). Initial growth of Moringa oleifera Lam. as a function of poultry litter doses and granulometry. Pesquisa Agropecuária Tropical, 48(4), 399-406. Recuperado de https://doi.org/10.1590/1983$40632018 v 4852447$.

Souza, L. M., Silva, M. M. A. \& Araújo, J. S. (2015). Aclimatização de mudas de palma forrageira: como fazer?. Campina Grande: INSA. Recuperado de https://portal.insa.gov.br/acervocartilhas/202-aclimatizacao-de-mudas-de-palma-forrageira-como-fazer.

Souza, M. (2003). Muito além do xaxim. Natureza, São Paulo, 182(2), 32-37.

Spangemberg, G. (2001). Progress in biotechnology offorage species: transgenies and genomics in molecular breeding of forage plants. Anais do Encontro Latinoamericano de Biotecnologia Vegetal, Goiânia, Brasil, 4.

Vieira, A. H., Azevedo, M. S. F. R., Rodrigues, V. G. S. \& Rossi, L. M. B. (1998). Efeito de diferentes substratos para producao de mudas de freijo-louro Cordia alliodora (Ruiz \& Pav.) Oken (Boletim de Pesquisa, v. 25). Porto Velho: Embrapa Rondônia. Recuperado de https://www.embrapa.br/rondonia/busca-de-publicacoes/-/publicacao/700019/efeito-dediferentes-substratos-para-producao-de-mudas-de-freijo-louro-cordia-alliodora-ruiz--pavoken. 
Villalobos, V. M. A. (2001). Aplicação do cultivo de tecidos para a micropropagação de Opuntia SP. In Agroecologia, cultivo e usos da palma forrageira (Estudos da FAO em Produção e Proteção Vegetal, 132). João Pessoa/PB: SEBRAE/PB.

Xavier, A., Wendling, I. \& Silva, R. L. (2009). Silvicultura clonal: princípios e técnicas. Viçosa: Editora UFV.

\section{COMO CITAR ESTE ARTIGO:}

Dutra, M. de F. B., Aloufa, M. H. I., Melo, N. F. de, Leite, J. I. P. (2020). Aclimatização de genótipos de palma forrageira Opuntia stricta (Haw.) E Nopalea cochenillifera (L.) Salm-Dyck resistentes a cochonilha-do-carmim (Dactylopius opuntiae). Holos. 36(7), 1-17.

\section{SOBRE OS AUTORES}

\section{DE F. B. DUTRA}

Bióloga, Mestre em Genética e Biologia Molecular e doutoranda do Programa de Desenvolvimento e Meio Ambiente da UFRN (PRODEMA) e colaboradora da Empresa de Pesquisa Agropecuária do Rio Grande do Norte ao qual participa de Projetos na área de Biotecnologia e atua como Coordenadora do Laboratório de Biotecnologia que pertence ao Governo do Estado do Rio Grande do Norte. E-mail: mfbdutra @ hotmail.com ORCID ID: http://orcid.org/0000-0002-5923-0674

\section{H. I. ALOUFA}

Doutorado em Biologia e Fisiologia Vegetal da UFRN, Professor e pesquisador lotado no Departamento de Botânica e Zoologia. Coordenador do Laboratório de Biotecnologia de Conservação de Espécies Nativas (LABCEN). E-mail: magdialoufal@gmail.com

ORCID ID: https://orcid.org/0000-0002-3762-9625

\section{N. F. DE MELO}

Biólogo pela Universidade Federal de Pernambuco (1990), Mestre em Botânica pela Universidade Federal Rural de Pernambuco (1994), Especialista em Biotecnologia Vegetal pelo Agricultural Biotechnology Centre/Hungria (1996) e Doutor em Ciências Biológicas (Área de concentração em Genética) pela Universidade Federal de Pernambuco (2002). Atua como pesquisador da Empresa Brasileira de Pesquisa Agropecuária (Embrapa Semiárido) e participa como professor permanente dos Programas de Pós-graduação em Recursos Genéticos Vegetais da Universidade Estadual de Feira de Santana - UEFS e do Mestrado Acadêmico em Agronomia - Produção Vegetal da Universidade Federal do Vale do São Francisco - Univasf. $\mathrm{Na}$ área de gestão, atuou como Chefe de Pesquisa e Desenvolvimento e Chefe-Geral da Embrapa Semiárido. Tem experiência na área de Biologia Aplicada, com ênfase em Biotecnologia e Citogenética Vegetal, sendo revisor de periódicos nacionais e internacionais, atuando principalmente nos seguintes temas: cultura in vitro de células e tecidos vegetais, micropropagação, micorriza, citogenética convencional e molecular e melhoramento genético vegetal. E-mail: natoniel.melo@embrapa.br

ORCID ID: $\quad$ https://orcid.org/0000-0001-6888-4090

\section{J. I. P. LEITE}

"IN MEMORIAM"

Possui graduação em Engenharia de Minas pela Universidade Federal da Paraíba (1987) e mestrado em 
Engenharia Química pela Universidade Federal do Rio Grande do Norte (1992). Atualmente é professor do Instituto Federal de Educação, Ciência e Tecnologia do Rio Grande do Norte. Tem experiência na área de Engenharia de Minas, com ênfase em Métodos de Concentração e Enriquecimento de Minérios, atuando principalmente nos seguintes temas: caracterização tecnológica, separação mineral, meio ambiente com ênfase em processamento mineral. E-mail: jypleite@gmail.com

ORCID ID: https://orcid.org/0000-0002-8331-697X

Editor(a) Responsável: Francinaide de Lima Silva Nascimento Pareceristas Ad Hoc: MAX PINHO E FRANCISCO CARVALHO MOREIRA

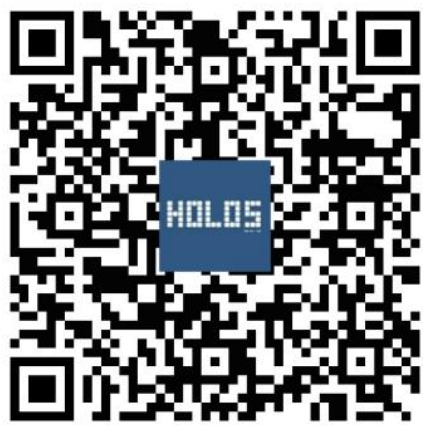

